\title{
Lack of correlation between short- and long-term performance after lung cancer surgery
}

Felix G. Fernandez, MD, MSc, ${ }^{a}$ Andrzej S. Kosinski, PhD, ${ }^{b}$ Betty C. Tong, MD, MHS, MS,

Anthony P. Furnary, MD, ${ }^{\mathrm{c}}$ Liqi Feng, MS, ${ }^{\mathrm{b}}$ Mark Onaitis, MD, ${ }^{\mathrm{d}}$ Daniel Boffa, MD,

Cameron D. Wright, MD, ${ }^{\mathrm{f}}$ Patricia Cowper, PhD, ${ }^{\mathrm{b}}$ Jeffrey P. Jacobs, MD, ${ }^{\mathrm{g}}$ Robert H. Habib, PhD, ${ }^{\mathrm{h}}$ and

Joe B. Putnam, MD

\section{ABSTRACT}

Objective: Outcomes for lung cancer surgery are currently measured according to perioperative morbidity and mortality. However, the oncologic efficacy of the surgery is reflected by long-term survival. We examined correlation between measures of short-term and long-term performance for lung cancer surgery.

Methods: The Society of Thoracic Surgeons General Thoracic Surgery Database linked to Medicare survival data was queried for pathologic stage I lung cancer resected between 2009 and 2013. Two separate multivariable models were created: (1) short-term: avoidance of perioperative major morbidity and mortality; and (2) long-term: 3-year survival. Standardized incidence ratios were calculated for the Society of Thoracic Surgeons programs (participants) to determine riskadjusted participant performance measures for the short- and long-term time points. Correlation of participant standardized incidence ratios for short- and long-term performance was assessed using the Pearson correlation coefficient.

Results: The study population included 12,596 patients from 229 participating programs. One hundred fifty-one participants met minimum volume and follow-up requirements for analysis. Overall, performance for the short-term measure was uniform with only $2(1.3 \%)$ participants performing better than expected and $2(1.3 \%)$ worse than expected. For the long-term measure, $9(6 \%)$ participants achieved better than expected and $5(3.3 \%)$ worse than expected survival. No participant was an above or below average performer for the short- and long-term measures. Further, no correlation was observed between participant short- and long-term performance (Pearson correlation coefficient, 0.12; 95\% confidence interval, -0.04 to $0.28 ; P=.14$ ).

Conclusions: Avoidance of perioperative morbidity and mortality is an incomplete measure of performance in lung cancer surgery. Lung cancer surgery performance metrics should assess the safety of surgery and long-term survival. (J Thorac Cardiovasc Surg 2019;157:1633-43)

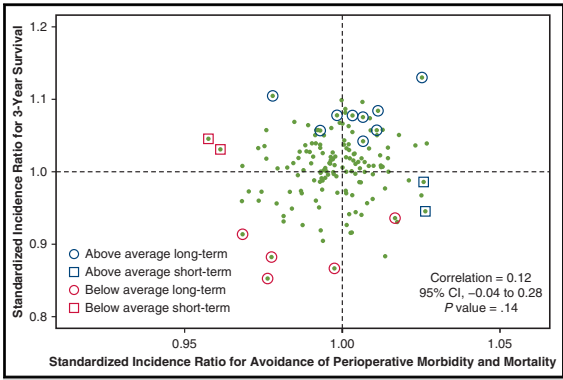

There was no correlation between short- and longterm performance measures for lung cancer surgery.

\section{Central Message}

Safety in lung cancer surgery, measured by avoidance of operative morbidity and mortality does not correlate with long-term survival. Safety of surgery as well as oncologic quality must be measured.

\section{Perspective}

Outcomes for lung cancer surgery are currently measured according to perioperative (shortterm) morbidity and mortality. However, the oncologic efficacy of the surgery is reflected by long-term survival. This study showed no correlation between measures of short- and long-term performance. Lung cancer surgery performance metrics should assess the safety of surgery as well as long-term survival.

See Commentaries on pages 1644 and 1646.
Clinical performance associated with lung cancer surgery is most frequently assessed according to the perioperative outcomes of morbidity and mortality. For example, the

\footnotetext{
From the ${ }^{\text {a}}$ Emory University School of Medicine, Atlanta, Ga; ${ }^{b}$ Duke University, Durham, NC; ${ }^{\mathrm{c}}$ Starr-Wood Cardiothoracic Group, Portland, Ore; ${ }^{\mathrm{d}}$ University of California San Diego, San Diego, Calif; ${ }^{\mathrm{e}}$ Yale University, New Haven, Conn; ${ }^{\mathrm{f}}$ Massachusetts General Hospital, Boston, Mass; ${ }^{\mathrm{g}}$ Johns Hopkins All Children's Hospi-

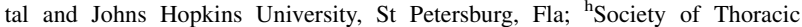
Surgeons Research Center, Chicago, Ill; and ${ }^{\mathrm{i} B a p t i s t ~ M . D . ~ A n d e r s o n ~ C a n c e r ~ C e n-~}$ ter, Jacksonville, Fla.

This project was supported by grant number R01 HS022279 from the Agency for Healthcare Research and Quality. The content is solely the responsibility of the authors and does not necessarily represent the official views of the Agency for Healthcare Research and Quality.
}

Society of Thoracic Surgeons (STS) General Thoracic Surgery Database (GTSD) participants have developed a risk model for lung cancer surgery and a composite score

\footnotetext{
Read at the 98th Annual Meeting of The American Association for Thoracic Surgery, San Diego, California, April 28-May 1, 2018.

Received for publication April 21, 2018; revisions received Sept 7, 2018; accepted for publication Sept 30, 2018; available ahead of print Jan 8, 2019.

Address for reprints: Felix G. Fernandez, MD, MSc, The Emory Clinic, 1365 Clifton Rd NE, Suite A2214, Atlanta, GA 30322 (E-mail: felix.fernandez@ emoryhealthcare.org).

$0022-5223 / \$ 36.00$

Copyright $(C) 2018$ by The American Association for Thoracic Surgery https://doi.org/10.1016/j.jtcvs.2018.09.141
} 


\section{Abbreviations and Acronyms \\ ASA = American Society of Anesthesiology \\ $\mathrm{CI}=$ confidence interval \\ $\mathrm{CMS}=$ Centers for Medicare and Medicaid Services \\ GTSD $=$ General Thoracic Surgery Database \\ SIR = standardized incidence ratio \\ STS = Society of Thoracic Surgeons \\ VATS $=$ video-assisted thoracic surgery}

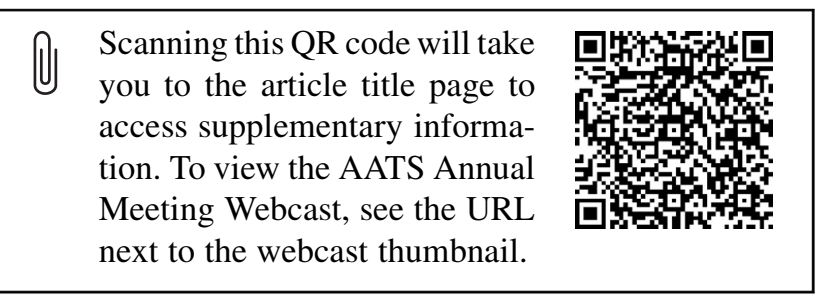

for rating program performance for lobectomy for lung cancer. ${ }^{1,2}$ Both use perioperative morbidity and mortality as the outcome measures. STS participants (programs participating in the database) are assigned a "star rating" of 1,2 , or 3 on the basis of their composite score for lobectomy as statistically below, not different, or above the STS mean, respectively. In the future, participants will have the opportunity to voluntarily publicly report their "star ratings" on the STS Web site.

Measures of perioperative morbidity and mortality assess the safety of an operation. However, these measures do not assess the completeness of surgical resection and pathologic staging, which affect long-term survival. The oncologic efficacy of a lung cancer surgery is reflected by the measures of cancer recurrence and long-term survival. However, challenges and time lag in collecting longitudinal recurrence and survival data on patients has limited their use as performance measures for lung cancer surgery.

Linkage of STS GTSD lung cancer operations with the Medicare database of the Centers for Medicare and Medicaid Services (CMS) has established longitudinal follow-up in a large cohort of STS patients. ${ }^{3}$ This linkage allows for the development of performance measures for lung cancer surgeries that assess long-term survival in STS GTSD participants. Our objective was to examine the correlation between risk-adjusted measures of short-term and long-term performance for lung cancer surgery in the STS-CMS linked cohort. Our hypothesis was that no correlation exists between these measures, because short-term outcomes are used to assess the safety of surgery and long-term survival the oncologic efficacy.

\section{METHODS \\ Institutional Review Board Approval}

The data used in this analysis represent a limited data set with no direct patient identifiers that was originally collected for nonresearch purposes. Therefore, the analysis of these data was declared by the Duke University Health System institutional review board to be research not involving human subjects and determined to be exempt from formal review (approval: Pro00055346; June 16, 2014).

\section{STS GTSD}

The STS GTSD was queried for all treatment-naive patients treated with surgical resection for pathologic stage I primary lung cancer from January 1, 2009 through December 31, 2013. This study encompassed 2 STS GTSD data collection instruments (v2.081 and v2.2), with various changes incorporated to reflect advancement in thoracic operations.

\section{Medicare Database of CMS and Data Linkage}

The CMS administrative claims data source for this study is the $100 \%$ Medicare Inpatient Claims file, which contains information on hospitalizations of patients enrolled in fee-for-service Medicare, and the Medicare Carrier Claims file, which contains physician billings. Also used was the $100 \%$ Medicare Master Beneficiary Summary file, which links to the inpatient file, contains information on beneficiary eligibility, demographic characteristics, and date of death. STS GTSD lung cancer surgery records were linked to CMS claims files using combinations of nonunique indirect identifiers through a deterministic matching algorithm, as previously described. ${ }^{3}$ Linkage to CMS data restricts the analysis to individuals age 65 years and older. Follow-up in the CMS data extends through the year 2013.

\section{Outcome Measures and Explanatory Variables}

The objective of this study was to examine whether correlation exists between risk-adjusted measures of short-term performance and long-term performance (survival) for lung cancer surgery. Therefore the primary outcome measures were: (1) avoidance of composite operative major morbidity and/or mortality (short-term measure); and (2) 3-year survival (long-term measure). Postoperative events were defined according the STS GTSD lung cancer resection risk model. ${ }^{1}$ Mortality during the index hospitalization for surgery or within 30 days of the procedure is classified as an operative mortality. Major morbidities were previously defined through empiric selection of important adverse outcomes. These include: tracheostomy, reintubation, initial ventilatory support for longer than 48 hours, adult respiratory distress syndrome, bronchopleural fistula, pulmonary embolus, pneumonia, unexpected return to the operating room, and myocardial infarction. Mortality is the most extreme complication but has a low event rate. The composite outcome was defined as having either operative mortality or at least 1 major morbidity. For long-term survival, date of death was determined from the CMS database for each patient whereas censorship was on the basis of the last month of CMS follow-up data available.

The following independent variables were adjusted for when examining short- and long-term outcomes: age, sex, body mass index, American Society of Anesthesiology risk class, Zubrod score, coronary artery disease, cerebrovascular disease, congestive heart failure, diabetes mellitus, steroid use, peripheral vascular disease, renal insufficiency, forced expiratory volume in 1 second percent predicted, smoking status (current, former, never), and whether or not the patient had a previous thoracic surgery that affects the operative field (ie, thoracic reoperation). The extent of resection (wedge, segment, lobe, etc) and operative approach (video-assisted thoracic surgery [VATS] vs thoracotomy) were noted but not adjusted for in the analyses. We recognize the potential for extent of resection to influence outcome. ${ }^{1}$ However, we consider extent of resection to be a 
component of the oncologic quality of the care provided by each participant and, therefore, should not be controlled for in the analysis. Several variables pertaining to the extent of the clinical staging evaluation in the 4 months before the surgery date were extracted from Medicare Carrier Claims files. These included claims for imaging (positron emission tomography and computed tomography scanning, brain magnetic resonance imaging, head computed tomography imaging) and invasive mediastinal staging (mediastinoscopy, endobronchial ultrasound, endoscopic ultrasound, thoracoscopy with mediastinal or pleural biopsy). Specific codes are listed in Appendix E1. 7.1\% of the study population did not have continuous Fee For Service Part A and B Medicare coverage for the 4 months preceding surgery, and therefore did not have data available for this analysis. The analysis was restricted to pathologic stage I lung cancer, because the long-term survival outcome would be most dependent on the completeness of resection and accuracy of pathologic staging rather than appropriate administration of and response to adjuvant therapies. Lung cancer pathologic stage was determined according to the American Joint Committee on Cancer seventh edition staging system.

The STS GTSD has been externally audited since $2010 .^{4}$ Audits have shown high agreement rates with hospital records and validated the accuracy and completeness of the data. Overall the rate of missing data was low (average of $3 \%$ across the data fields studied). The greatest number of instances of missing data was for the variable "forced expiratory volume in 1 second percent predicted" (missing in approximately $10 \%$ of patients). For a number of variables (comorbidities and whether or not the surgery represented a reoperation), failure to code the presence of a variable was considered to be a negative response. The rate of no response ranged from $0.2 \%$ (smoking status) to $7.6 \%$ (perioperative steroids). Model refinement for regression models were limited to cases that were complete for all data points considered (implied negative responses considered complete).

\section{Statistical Analyses}

Descriptive analyses were presented as counts with percentages. Differences between groups were assessed using $\chi^{2}$ tests. A multivariable logistic regression generalized mixed model was created to estimate the association of patient baseline characteristics with the primary outcome measure of avoidance of composite operative major morbidity and mortality (shortterm measure). A gamma frailty survival model was used for long-term survival and estimation of 3-year survival (long-term measure). ${ }^{5}$ Clustering of patients within participants (programs) was accounted for in the both models by considering participants as random effects. A Bayesian framework was used. Operative mortality and patients with not more than 60 days of follow-up were excluded from the long-term survival analysis (see Appendix E2, Consolidated Standards of Reporting Trials flow diagram). All covariates were retained in the models.

We examined variation in participant performance for the outcomes of composite operative major morbidity or mortality and 3-year survival. We estimated Bayesian $90 \%$ credible intervals for standardized incidence ratio (SIR) for each GTSD participant for the short-term and long-term outcome measures (additional detail on Bayesian methodology provided in Appendix E3). Ninety percent credible intervals were used rather than $95 \%$ credible intervals, as used in the STS composite score for lobectomy. ${ }^{2}$ Such an approach was adopted to liberalize the identification of outliers only for purposes of this analysis. Even though all of the available patients were used for the multivariable models, participants performing fewer than 10 lung cancer resections per year and/or had fewer than 10 linked cases or had all patients followed fewer than 3 years were excluded from the SIRbased presentation and analysis. SIRs summarize participant performance, as previously described. ${ }^{1,6}$ The SIR is the ratio between the participant's risk-adjusted rate and the risk-adjusted rate of a hypothetical average GTSD participant. For the short-term outcome in this study, a SIR $>1.0$ represents a higher risk-adjusted freedom from composite operative major morbidity or mortality compared with an average participant. For the long-term outcome in this study, a SIR $>1.0$ is consistent with a higher risk-adjusted 3-year survival compared with an average participant. Finally, the correlation of program SIRs for short- and long-term performance was assessed using the Pearson correlation coefficient. Analyses were run using the SAS software version 9.4 (SAS Institute, Cary, NC) and $\mathrm{R}$ software version 3.1.2. ${ }^{7}$ A $P$ value of $<.05$ was considered to be significant.

\section{RESULTS}

The study population included 12,596 patients from 229 participating programs, with 151 participants fulfilling minimum volume and follow-up requirements for analysis of participant SIR-based performance. Descriptive characteristics of the patient population are shown in Table 1. The operative mortality for patients who underwent lung cancer surgery in the study cohort was $1.6 \%$. The frequency of each of the major morbidities is shown in Table 2. Estimated overall 3-year survival was $77.8 \%$ for the entire cohort.

SIRs were calculated with $90 \%$ Bayesian credible intervals to determine the distribution of performance among STS GTSD participants for the composite measure of avoidance of major morbidity and mortality (short-term performance; Figure 1). There was no overlap in credible intervals between some of the best $(1.3 \%$ for 2 of 151 sites with lower limit $>1)$ and worst performing sites $(1.3 \%$ for 2 of 151 sites with upper limit $<1$ ), indicating that our model provides meaningful discrimination between best and worst performers. Overall, participants in the STS GTSD showed similar perioperative outcomes, because only $1.3 \%$ performed better than expected and $1.3 \%$ worse than expected (c-statistic for model, 0.69). Perioperative major morbidity rates for above average, average, and below average performers were $4.2 \%, 8.9 \%$, and $15.8 \%$, respectively, whereas mortality rates were $1.9 \%, 2.9 \%$, and $3.9 \%$, respectively.

Figure 2 shows participant SIRs with $90 \%$ Bayesian credible intervals for the long-term measure of survival at 3 years (long-term performance). Greater variability was observed compared with short-term SIRs. Again, there was no overlap in credible intervals between some of the best $(6 \%$ for 9 of 151 sites with lower limit $>1)$ and worst performing sites $(3.3 \%$ for 5 of 151 sites with upper limit $<1$ ), indicating that this model provides meaningful discrimination between best and worst performers for long-term survival (c-statistic for model, 0.674). Riskadjusted survival curves for high and low performers are shown in Figure 3. Differences in 3-year survival between above average, average, and below average participants were clinically meaningful at $88.1 \%, 78.8 \%$, and $66.6 \%$, respectively.

We next examined a scatter plot of participant SIRs for the short- and long-term outcome measures, as shown in Figure 4. No participants were high or low performers for the short- and long-term performance measures. Examination using the Pearson correlation coefficient $(0.12 ; 95 \%$ 
TABLE 1. Patient characteristics

\begin{tabular}{|c|c|}
\hline Characteristic & n ( $\%$ of all patients) \\
\hline \multicolumn{2}{|l|}{ Age, $y$} \\
\hline $65-69$ & $3672(29.2)$ \\
\hline $70-74$ & $3757(29.8)$ \\
\hline $75-79$ & $3010(23.9)$ \\
\hline 80 and older & $2157(17.1)$ \\
\hline \multicolumn{2}{|l|}{ Sex } \\
\hline Male & $5682(45.1)$ \\
\hline Female & $6914(54.9)$ \\
\hline \multicolumn{2}{|l|}{ BMI } \\
\hline$<18.5$ & $293(2.3)$ \\
\hline $18.5-25$ & $4114(32.7)$ \\
\hline $25-30$ & $4929(39.1)$ \\
\hline $30-35$ & $2238(17.8)$ \\
\hline$>35$ & $1022(8.1)$ \\
\hline \multicolumn{2}{|l|}{ ASA risk class } \\
\hline I-II & $1553(12.3)$ \\
\hline III & 9654 (76.6) \\
\hline IV-V & $1389(11.0)$ \\
\hline \multicolumn{2}{|l|}{ Zubrod score } \\
\hline 0 & $5650(44.9)$ \\
\hline 1 & $6404(50.8)$ \\
\hline $2-5$ & $542(4.3)$ \\
\hline CAD & $3372(26.8)$ \\
\hline CVD & $1251(9.9)$ \\
\hline $\mathrm{CHF}$ & $445(3.5)$ \\
\hline DM & $2443(19.4)$ \\
\hline Steroid use & $416(3.3)$ \\
\hline PVD & $1500(11.9)$ \\
\hline Renal insufficiency & $269(2.1)$ \\
\hline \multicolumn{2}{|l|}{ FEV1 \% predicted } \\
\hline$>80$ & $6653(52.8)$ \\
\hline $60-80$ & $3856(30.6)$ \\
\hline $40-60$ & $1772(14.1)$ \\
\hline$<40$ & $315(2.5)$ \\
\hline \multicolumn{2}{|l|}{ Cigarette use } \\
\hline Never & $1724(13.7)$ \\
\hline Past & $8623(68.5)$ \\
\hline Current & $2249(17.9)$ \\
\hline Thoracic reoperation procedure & $688(5.5)$ \\
\hline Lobectomy & $8507(67.5)$ \\
\hline Bilobectomy & $206(1.6)$ \\
\hline Pneumonectomy & $83(0.7)$ \\
\hline Segmentectomy & $1131(9.0)$ \\
\hline Sleeve lobectomy & $73(0.6)$ \\
\hline Wedge resection & $2596(20.6)$ \\
\hline VATS approach & $7943(63.1)$ \\
\hline
\end{tabular}

BMI, Body mass index; $A S A$, American Society of Anesthesiology; CAD, coronary artery disease; $C V D$, cerebrovascular disease; $C H F$, congestive heart failure; $D M$, diabetes mellitus; $P V D$, peripheral vascular disease; $F E V 1$, forced expiratory volume in 1 second; VATS, video-assisted thoracic surgery.
TABLE 2. Frequency of major morbidities

\begin{tabular}{lc}
\hline \multicolumn{1}{c}{ Complication } & n (\% of all patients) \\
\hline Pneumonia & $474(3.8)$ \\
Unexpected return to OR & $439(3.5)$ \\
Reintubation & $399(3.2)$ \\
Tracheostomy & $105(0.8)$ \\
ARDS & $73(0.6)$ \\
Initial ventilation $>48$ h & $55(0.4)$ \\
Pulmonary embolus & $55(0.4)$ \\
Myocardial infarction & $51(0.4)$ \\
Bronchopleural fistula & $43(0.3)$ \\
Mortality & $199(1.6)$ \\
Operative mortality & (30-day + in-hospital) \\
$\quad$ 90-Day mortality & $360(2.9)$ \\
\hline$O R$, Operating room, $A R D S$, adult respiratory distress syndrome
\end{tabular}

confidence interval $[\mathrm{CI}],-0.04$ to $0.28 ; P=.14$ ) showed no correlation between participant short- and long-term performance, measured according to the respective SIRs. As a sensitivity analysis, we also examined correlation between short- and long-term performance while adjusting for extent of resection (procedure type) in the calculation of shortterm SIRs. Again, no correlation was noted with the Pearson correlation coefficient $(0.12 ; 95 \% \mathrm{CI},-0.05$ to 0.27 ; $P=.16)$. Finally, the analysis was also restricted to only lobectomies and again no correlation was observed $(0.03$; $95 \% \mathrm{CI},-0.13$ to $0.20 ; P=.70)$.

Descriptive characteristics of above average, average, and below average performers with respect to the longterm performance measure are presented in Table 3. It is notable that better than expected performers operated on fewer current smokers compared with average and lower than average performers. It is also interesting to note that high performers used VATS approaches and performed lobectomies less frequently than average or low performers. However, high performers did use segmental resections more often. Finally, invasive mediastinal staging also was performed more frequently by above average participants.

\section{DISCUSSION}

This study shows that lung cancer surgery performed by STS participants is safe and consistent. In addition, resection of pathologic stage I lung cancers results in acceptable long-term survival. We were able to identify statistically different high and low performing outliers for short-term and long-term lung cancer surgery performance measures. However, we found no correlation between participant short- and long-term performance measures, measured 


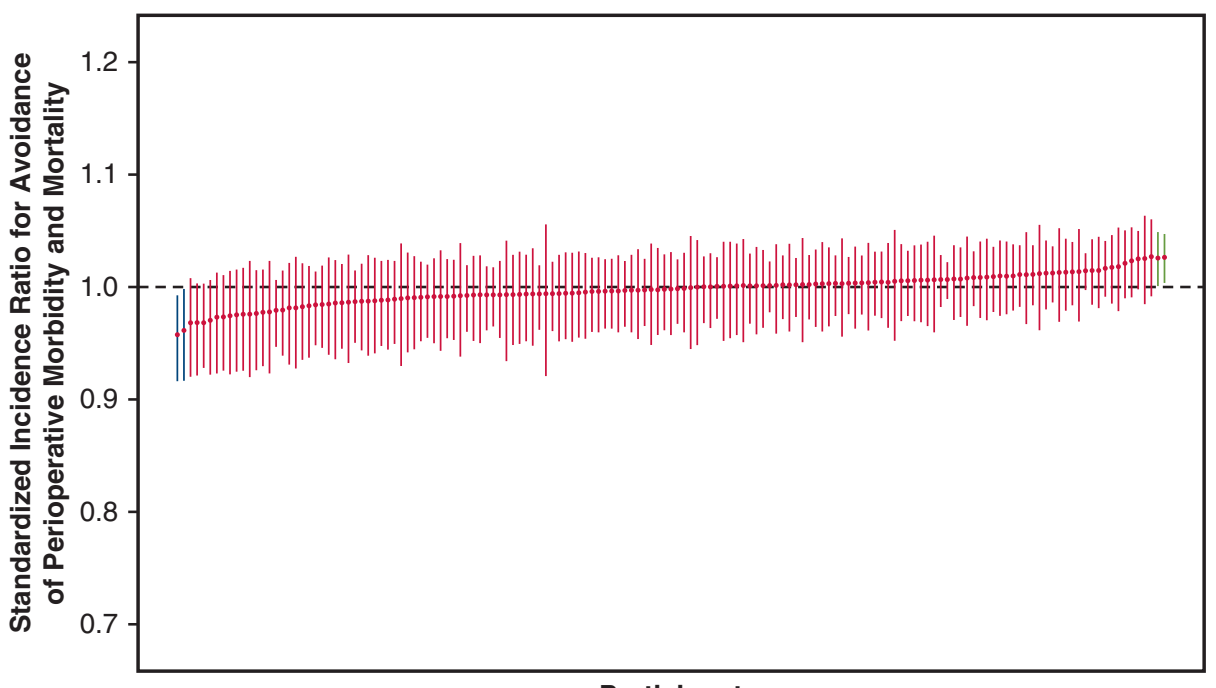

Participant

FIGURE 1. Participant performance variability for avoidance of the composite of major morbidity and mortality (short-term measure). Standardized incidence ratios with $90 \%$ Bayesian credible intervals are shown.

according to the SIRs derived in this study. These findings raise concern that measurement of perioperative safety outcomes alone might not completely identify quality in surgical therapy for lung cancer.

The STS has developed a multidimensional composite score for the measurement of participant performance for lobectomy for lung cancer. ${ }^{2}$ The outcomes that comprise this performance measure are risk-adjusted operative mortality and any-or-none risk-adjusted major morbidity. Using 95\% Bayesian credible intervals, participants are identified as above average, average, and below average relative to the STS participant mean and given a "star rating." This measure is used for quality assessment and provider feedback.
The "star rating" will be made available for voluntary public reporting. The STS has also developed a risk model for lung cancer resection that is inclusive of all extents of resection for lung cancer (wedge resection through pneumonectomy). ${ }^{1}$ This model is used to risk-adjust surgical outcomes and to derive participant SIRs. The outcome measures are also operative mortality and any-or-none major morbidity. The composite score and risk model have been shown to identify differences in perioperative performance and outcomes among STS participants. In our study, we showed that lung cancer surgery performed by STS participants is safe and consistent with little variability in perioperative outcomes and few outliers identified.

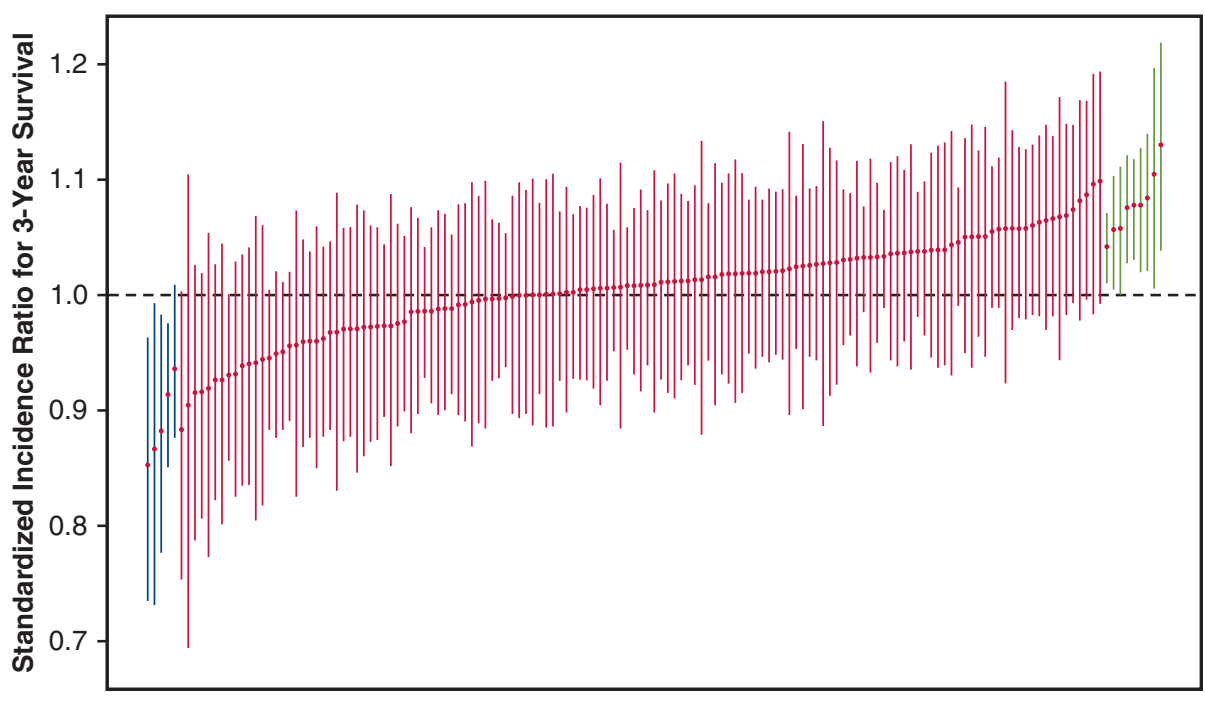

Participant

FIGURE 2. Participant performance variability for conditional 3-year survival (long-term measure). Standardized incidence ratios with $90 \%$ Bayesian credible intervals are shown. 


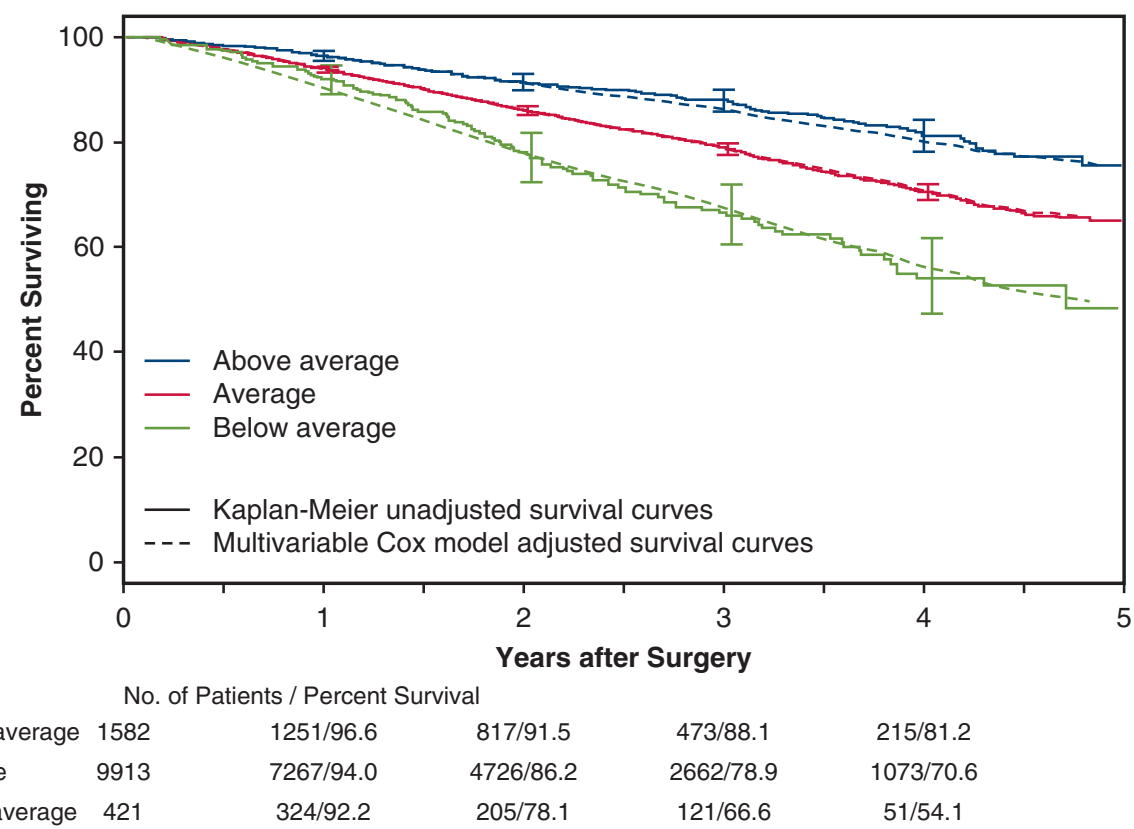

FIGURE 3. Kaplan-Meier unadjusted survival curves and multivariable Cox model adjusted survival curves for pathologic stage I lung cancer in above average, average, and below average participants.

Unfortunately, it is possible to perform a safe lung cancer operation that is of low quality. For instance, a surgeon could incompletely resect a primary lung cancer leaving a positive margin and perform no lymph node evaluation (sampling or dissection) potentially failing to identify occult nodal metastatic disease. Such a patient would be at high risk for local, regional, and systemic recurrence and would not be offered adjuvant chemotherapy, as would be indicated if the nodal disease was identified. ${ }^{8}$ Such a patient would be expected to have a poor prognosis with respect to long-term survival. However, if the surgeon performed this operation safely, without the occurrence of perioperative morbidity and mortality, this operation would be considered a quality operation and improve the participant's performance rating. Linden and colleagues have previously shown that wedge resections for lung cancer have a $37 \%$ lower mortality rate and a $50 \%$ lower major morbidity rate compared with anatomic resections among STS GTSD participants. ${ }^{9}$ However, our previous work with STS-CMS linked data showed that wedge resections are

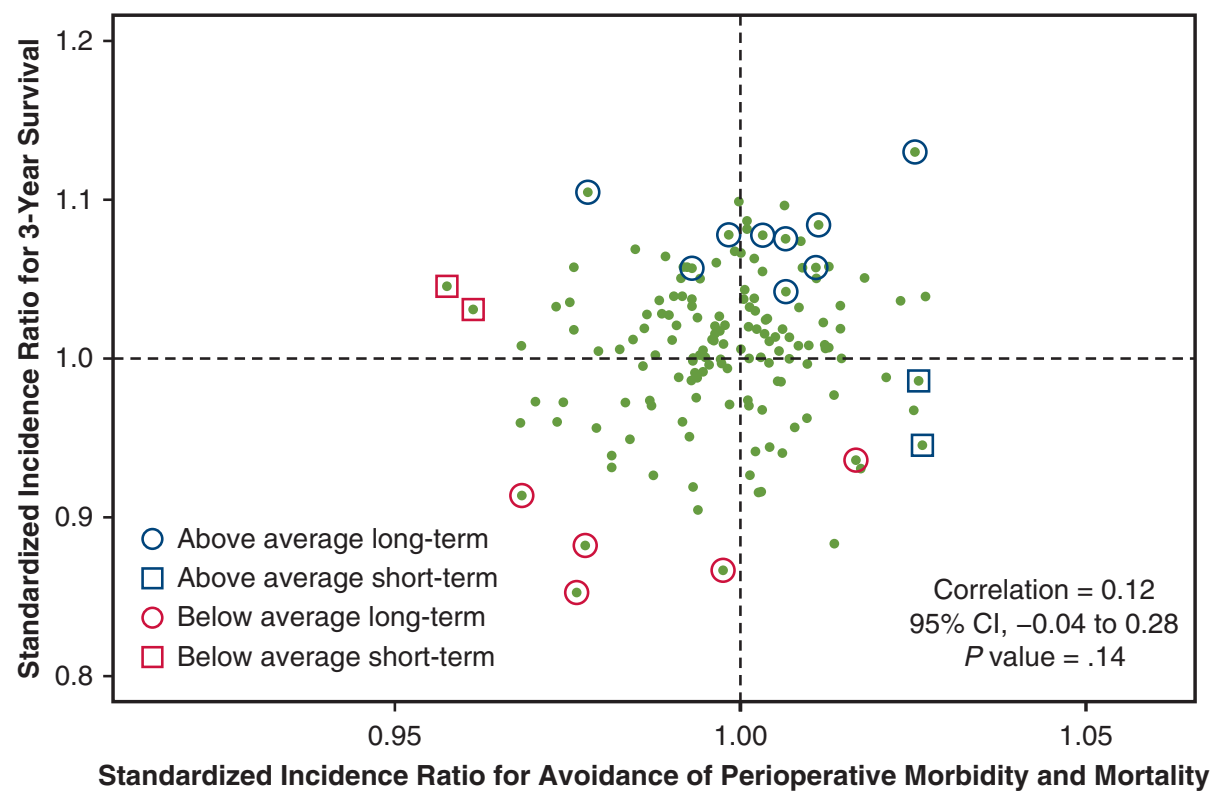

FIGURE 4. Scatter plot of participant standardized incidence ratios for the short-term and long-term performance measures. $C I$, Confidence interval. 
TABLE 3. Patient characteristics for above average, average, and below average performance for long-term performance measure

\begin{tabular}{|c|c|c|c|c|}
\hline Characteristic & $\begin{array}{c}\text { Above average, } \\
\text { n }(\%)\end{array}$ & $\begin{array}{c}\text { Average, } \\
\text { n (\%) }\end{array}$ & $\begin{array}{c}\text { Below average, } \\
\text { n }(\%)\end{array}$ & $P$ value \\
\hline Age (y) & & & & .96 \\
\hline $65-69$ & 465 (29.4) & 2938 (29.6) & $121(28.7)$ & \\
\hline $70-74$ & $479(30.3)$ & 2957 (29.8) & $130(30.9)$ & \\
\hline $75-79$ & $370(23.4)$ & 2346 (23.7) & $106(25.2)$ & \\
\hline 80 and older & 268 (16.9) & $1672(16.9)$ & $64(15.2)$ & \\
\hline Sex & & & & .37 \\
\hline Male & $686(43.4)$ & 4475 (45.1) & $184(43.7)$ & \\
\hline Female & $896(56.6)$ & $5438(54.9)$ & $237(56.3)$ & \\
\hline BMI & & & & .047 \\
\hline$<18.5$ & $25(1.6)$ & $237(2.4)$ & $11(2.6)$ & \\
\hline $18.5-25$ & $518(32.7)$ & $3205(32.3)$ & 154 (36.6) & \\
\hline $25-30$ & $598(37.8)$ & 3952 (39.9) & $143(34.0)$ & \\
\hline $30-35$ & 305 (19.3) & $1716(17.3)$ & $82(19.5)$ & \\
\hline$>35$ & $136(8.6)$ & $803(8.1)$ & $31(7.4)$ & \\
\hline ASA risk class & & & & $<.001$ \\
\hline I-II & $402(25.4)$ & $1026(10.4)$ & $71(16.9)$ & \\
\hline III & 1059 (66.9) & $7766(78.3)$ & $312(74.1)$ & \\
\hline IV-V & $121(7.6)$ & $1121(11.3)$ & $38(9.0)$ & \\
\hline Zubrod score & & & & $<.001$ \\
\hline 0 & $730(46.1)$ & 4408 (44.5) & $241(57.2)$ & \\
\hline 1 & $794(50.2)$ & $5088(51.3)$ & $160(38.0)$ & \\
\hline $2-5$ & $58(3.7)$ & $417(4.2)$ & $20(4.8)$ & \\
\hline CAD & $416(26.3)$ & $2603(26.3)$ & $114(27.1)$ & .93 \\
\hline CVD & $154(9.7)$ & $959(9.7)$ & 47 (11.2) & .60 \\
\hline $\mathrm{CHF}$ & $41(2.6)$ & $340(3.4)$ & $22(5.2)$ & .024 \\
\hline $\mathrm{DM}$ & 297 (18.8) & $1917(19.3)$ & $92(21.9)$ & .36 \\
\hline Steroid use & $48(3.0)$ & $311(3.1)$ & $18(4.3)$ & .40 \\
\hline PVD & $180(11.4)$ & 1135 (11.4) & $75(17.8)$ & $<.001$ \\
\hline Renal insufficiency & $30(1.9)$ & $200(2.0)$ & $15(3.6)$ & .081 \\
\hline FEV1 \% predicted & & & & .29 \\
\hline$>80$ & 877 (55.4) & $5211(52.6)$ & $215(51.1)$ & \\
\hline $60-80$ & $450(28.4)$ & 3065 (30.9) & $127(30.2)$ & \\
\hline $40-60$ & $220(13.9)$ & $1387(14.0)$ & $69(16.4)$ & \\
\hline$<40$ & $35(2.2)$ & $250(2.5)$ & $10(2.4)$ & \\
\hline Cigarette use & & & & $<.001$ \\
\hline Never & $226(14.3)$ & $1371(13.8)$ & 47 (11.2) & \\
\hline Past & $1139(72.0)$ & $6735(67.9)$ & $286(67.9)$ & \\
\hline Current & 217 (13.7) & $1807(18.2)$ & $88(20.9)$ & \\
\hline Thoracic reoperation & $76(4.8)$ & $523(5.3)$ & $35(8.3)$ & .015 \\
\hline Procedure & & & & $<.001$ \\
\hline Lobectomy & $997(63.0)$ & $6768(68.3)$ & $273(64.8)$ & \\
\hline Bilobectomy & $11(0.7)$ & $172(1.7)$ & $4(1.0)$ & \\
\hline Pneumonectomy & $10(0.6)$ & $61(0.6)$ & $5(1.2)$ & \\
\hline Segmentectomy & $181(11.4)$ & $862(8.7)$ & $31(7.4)$ & \\
\hline Sleeve lobectomy & $9(0.6)$ & $60(0.6)$ & $2(0.5)$ & \\
\hline Wedge resection & $374(23.6)$ & $1990(20.1)$ & $106(25.2)$ & \\
\hline VATS approach & $939(59.4)$ & $6271(63.3)$ & $312(74.1)$ & $<.001$ \\
\hline \multicolumn{5}{|l|}{ Preoperative staging } \\
\hline CT scan & $1340(92.4)$ & $8328(90.3)$ & $371(94.6)$ & $<.001$ \\
\hline
\end{tabular}


TABLE 3. Continued

\begin{tabular}{|c|c|c|c|c|}
\hline Characteristic & $\begin{array}{c}\text { Above average, } \\
\text { n (\%) }\end{array}$ & $\begin{array}{c}\text { Average, } \\
\text { n }(\%)\end{array}$ & $\begin{array}{c}\text { Below average, } \\
\text { n }(\%)\end{array}$ & $P$ value \\
\hline PET scan & $1200(82.8)$ & $7759(84.1)$ & $327(83.4)$ & .40 \\
\hline Brain imaging & $582(40.1)$ & $2604(28.2)$ & 86 (21.9) & $<.001$ \\
\hline Invasive mediastinal staging & $532(36.7)$ & $2496(27.1)$ & $129(32.9)$ & $<.001$ \\
\hline
\end{tabular}

$B M I$, Body mass index; $A S A$, American Society of Anesthesiology; $C A D$, coronary artery disease; $C V D$, cerebrovascular disease; $C H F$, congestive heart failure; $D M$, diabetes mellitus; $P V D$, peripheral vascular disease; FEVI, forced expiratory volume in 1 second; VATS, video-assisted thoracic surgery; $C T$, computed tomography; $P E T$, positron emission tomography.

associated with significantly worse long-term survival compared with lobectomy. ${ }^{10}$

Clearly, measures of oncologic quality are needed to assess performance in lung cancer surgery. Available outcome measures include recurrence-free survival, disease-free survival, and overall survival. Because tumor recurrence and cause of death are more difficult to ascertain and capture, risk-adjusted stage-specific overall survival is the most practical outcome measure to use. Process measures such as guideline-concordant preoperative staging, completeness of resection, use of anatomic resection, and measures of lymph node evaluation might serve as surrogates for the quality of the oncologic resection. ${ }^{11,12}$ However, there exist challenges with the definitions and/ or measurement of each of these process measures.

The absence of long term follow-up beyond in-hospital or 30 days in the STS GTSD has precluded the development of performance metrics for long-term survival. By establishing a linkage to CMS data, we are now able to examine survival in elderly patients who undergo lung cancer surgery. ${ }^{3}$ Our group has previously developed a risk model for longterm survival after surgical resection of lung cancer which is on the basis of patient, disease, and treatment characteristics. ${ }^{10}$ In the current study we found that risk-adjusted expected 3-year survival varied across STS participants, with high and low performers identified. The analysis was restricted to pathologic stage I lung cancers, because the risk-adjusted long-term survival would be most attributable to the completeness of resection and accuracy of staging. This analysis was not confounded by whether or not guideline-appropriate adjuvant chemotherapy was administered. ${ }^{13}$ As noted, no participants were either positive outliers or negative outliers for the short- and long-term performance measures despite the fact that the identification of outliers was liberalized with $90 \%$ credible intervals. That no correlation was observed among participant shortand long-term SIRs implies that the safety of lung cancer surgery and the quality of the oncologic resection are not coupled and need to be measured independently.

Attributes of the above average performing participants for the long-term measure were identified. Above average performers operated on patients with less comorbidities and on fewer current smokers compared with average and below average performers. Smoking status has consistently been identified as being associated with worse short- and long-term outcome after lung cancer surgery. ${ }^{1,10}$ A greater prevalence of never smokers in the above average group could be a marker for a greater prevalence of minimally invasive adenocarcinomas, which would be associated with better survival. Invasive mediastinal staging was also performed with greater frequency in above average participants, suggesting that observed survival differences might be attributable to the accuracy of staging. Use of invasive mediastinal staging has previously been associated with improved survival in patients who undergo lung cancer surgery. ${ }^{12}$ It is also interesting to note that high performers used VATS less frequently than average or low performers. Although this finding is unexpected, it potentially might be explained by other unmeasured confounders including: completeness of resection, thoroughness of nodal staging, and other center factors. The alternative explanation, that VATS is an inferior oncologic operation, is in contrast to the findings of several other studies. $^{14,15}$

There are several important limitations to consider with respect to this study. First, although the analysis was restricted to pathologic stage I lung cancer, long-term survival might be dependent on many other patient, health system, and societal factors that are out of the surgeon's control. This is likely why more variability was observed with the long-term SIRs compared with the short-term SIRs in our analysis. Second, we only considered operative morbidity and mortality and long-term survival as outcome measures. Quality of life is of significant importance to patients, and our data do not contain patient-reported outcomes. Future incorporation of patient-reported outcomes into the STS GTSD will address this limitation. ${ }^{16}$ Some variables such as diffusing capacity of the lung for carbon monoxide could not be included because of missing data and others are not captured (eg, frailty). Further, comorbidities are recorded as binary rather than graded. Additionally, a small percentage of the pathologic stage I lung cancer patients would be eligible for the receipt of adjuvant chemotherapy, but these data could not be captured. These data are also limited to patients 65 years of age or older with Medicare insurance who received care at centers participating in the STS GTSD, which is thought to represent approximately one-third of all patients who undergo lung cancer resection nationally. It is possible that the participant performance identified in this study for short- and long-term 
outcomes might be different in a younger patient population. The STS GTSD has recently begun to capture longterm survival data on all lung cancer surgeries. Finally, lung cancer surgery outcomes reported in the GTSD have been shown to be superior to those reported from national administrative databases, and the findings might not be generalizable to patients who undergo lung cancer resection by non-STS GTSD participants. ${ }^{17}$

A reasonable argument can be made that it is difficult to hold surgeons accountable to a benchmark survival result. Longitudinal data are difficult to collect. The time lag in collecting the data means that outcomes might not reflect care currently being provided by a surgeon or center. Also, the there are several unmeasured confounders that might affect long-term survival. Future work will focus on determining those process measures (preoperative staging evaluation, completeness of resection, and intraoperative lymph node evaluation, etc) that are most associated with survival. Such data, although also fraught with challenges, might provide more timely feedback on the oncologic quality of a lung cancer surgery and complement long-term survival outcomes.

\section{CONCLUSIONS}

Current performance metrics in lung cancer surgery address the safety of surgery but not oncologic quality. The absence of performance measures focused on longterm outcomes is an existing gap. Quality and performance measurement in lung cancer surgery should consider the avoidance of perioperative morbidity and mortality as well as long-term survival. Our future efforts will focus on development of performance metrics that incorporate both of these important perspectives.

\section{Webcast}

You can watch a Webcast of this AATS meeting presentation by going to: https://aats.blob.core.windows.net/ media/18Apr29/25ABC\%20General\%20Thoracic\%20SS/ S53/S53_7_webcast_045147494.mp4.

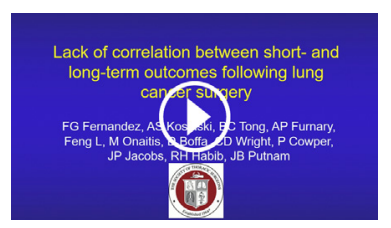

\section{Conflict of Interest Statement}

Authors have nothing to disclose with regard to commercial support.

\section{References}

1. Fernandez FG, Kosinski AS, Burfeind W, Park B, DeCamp MM, Seder C, et al. The Society of Thoracic Surgeons lung cancer resection risk model: higher quality data and superior outcomes. Ann Thorac Surg. 2016;102:370-7.

2. Kozower BD, O’Brien SM, Kosinski AS, Magee MJ, Dokholyan R, Jacobs JP, et al. The Society of Thoracic Surgeons composite score for rating program performance for lobectomy for lung cancer. Ann Thorac Surg. 2016;101:1379-87.

3. Fernandez FG, Furnary AP, Kosinski AS, Onaitis MW, Kim S, Boffa D, et al. Longitudinal follow-up of lung cancer resection from the Society of Thoracic Surgeons general thoracic surgery database in patients 65 years and older. Ann Thorac Surg. 2016;101:2067-76.

4. Magee MJ, Wright CD, McDonald D, Fernandez FG, Kozower BD. External validation of the Society of Thoracic Surgeons general thoracic surgery database. Ann Thorac Surg. 2013;96:1734-9.

5. Therneau TM, Grambsch PM. Modeling Survival Data: Extending the Cox Model. New York: Springer-Verlag; 2000.

6. Kozower BD, Sheng S, O'Brien SM, Liptay MJ, Lau CL, Jones DR, et al. STS database risk models: predictors of mortality and major morbidity for lung cancer resection. Ann Thorac Surg. 2010;90:875-83.

7. R Core Team. R: A Language and Environment for Statistical Computing. Vienna, Austria: R Foundation for Statistical Computing; 2014. Available at: http://www.R-project.org/. Accessed December 5, 2017.

8. Winton T, Livingston R, Johnson D, Rigas J, Johnston M, Butts C, et al. Vinorelbine plus cisplatin vs. observation in resected non-small cell lung cancer. $N$ Engl J Med. 2005;352:2589-97.

9. Linden PA, D'Amico TA, Perry Y, Saha-Chaudhuri P, Sheng S, Kim S, et al Quantifying the safety benefits of wedge resection: a Society of Thoracic Surgery database propensity-matched analysis. Ann Thorac Surg. 2014;98:1705-11.

10. Onaitis MW, Furnary AP, Kosinski AS, Kim S, Boffa D, Tong BC, et al. Prediction of long-term survival after lung cancer surgery for elderly patients in The Society of Thoracic Surgeons general thoracic surgery database. Ann Thorac Surg. 2018:105:309-16.

11. Samson P, Crabtree T, Broderick S, Kreisel D, Krupnick AS, Patterson GA, et al Quality measures in clinical stage I non-small cell lung cancer: improved performance is associated with improved survival. Ann Thorac Surg. 2017;103:303-11.

12. Farjah F, Flum DR, Ramsey SD, Heagerty PJ, Symons RG, Wood DE Multi-modality mediastinal staging for lung cancer among Medicare beneficiaries. J Thorac Oncol. 2009;4:355-63.

13. Ettinger D, Johnson B. Update: NCCN small cell and non-small cell lung cance clinical practice guidelines. J Natl Compr Canc Netw. 2005;3(suppl 1):S17-21.

14. Berry MF, D'Amico TA, Onaitis MW, Kelsey CR. Thoracoscopic approach to lobectomy for lung cancer does not compromise oncologic efficacy. Ann Thorac Surg. 2014;98:197-202.

15. Yang CJ, Kumar A, Klapper JA, Hartwig MG, Tong BC, Harpole DH Jr, et al. A national analysis of long-term survival following thoracoscopic versus open lobectomy for stage I non-small-cell lung cancer. Ann Surg. 2019;269: 163-71.

16. Khullar OV, Rajaei MH, Force SD, Binongo JN, Yi Lasanajak Y, Robertson S, et al. Pilot study to integrate patient reported outcomes after lung cancer surgery into the STS database. Ann Thorac Surg. 2017;104:245-53.

17. LaPar DJ, Bhamidipati CM, Lau CL, Jones DR, Kozower BD. The Society of Thoracic Surgeons general thoracic surgery database: establishing generalizability to national lung cancer resection outcomes. Ann Thorac Surg. 2012;94:216-21.

Key Words: lung cancer surgery, outcomes

\section{Discussion}

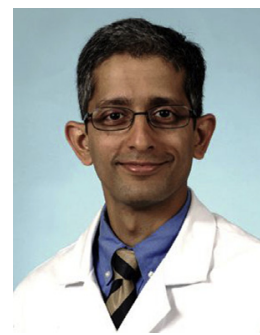
the STS General Thoracic Database presents before all of 
us who may be NPR-avid listeners "The Lake Wobegon effect," where all the children are above average. When you compare the STS outcomes with outcomes from other national large databases, the outcomes are superior in almost every aspect of evaluation. So that's one thing that I would point all listeners to and readers of the papers, to understand that we are representing better performers than national surgical programs that deal with lung cancer.

Two brief questions, which I will pose one after the other. In the data set you utilized pathologic stage I disease as the entry criterion for analysis. How and if at all in what way do you think things would have changed if you had used clinical stage I disease as the entry criterion for analysis?

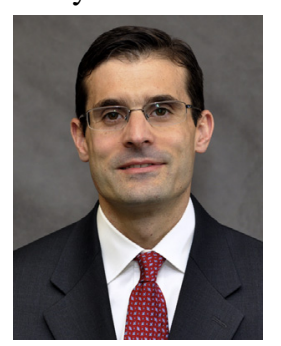

Dr Felix Fernandez (Atlanta, Ga). Thank you for your comment and question, Varun. That is a good question and we thought about it a lot. We selected pathologic stage I lung cancer because the outcome would be most dependent on the completeness of resection and accuracy of staging. If you studied clinical stage I lung cancer, then you would have some patients upstaged and then you take into account the variable of whether they received adjuvant chemotherapy and was it appropriate adjuvant chemotherapy. So we selected pathologic stage I lung cancer.

Having said that, we performed many sensitivity analyses in this study, and one was to do it with clinical stage I lung cancer, and we reached the same conclusion.

Dr Puri. Very good. Second, the current STS quality metrics, which you showed in your first slide, are representative of only short-term outcomes after major lung resection. In light of your findings, how do you feel that those metrics should be changed or modified either with immediate or subsequently long-term effect to those guidelines?

Dr Fernandez. That's another very important question. We do need to measure the safety of the operation, it's important, but as has been demonstrated in our study and many others that lung cancer surgery is fairly safe across a variety of programs, and if you look at the standardized incidence ratios, the confidence intervals are tight and there is not a lot of variability. The long-term survival was shown on the same scale as the figure with the short-term SIRs.

How we start to incorporate measures of oncologic qualities is a little more challenging. Should we hold surgeons accountable to a benchmark long-term survival metric that is not completely under their control and would reflect what happened at their institution 3 years or 5 years in the past? That's challenging.

You have published work on oncologic quality indicators: guideline-concordance staging, completeness of resection, anatomic resection, some measure of lymph node assessment. These are all fraught with problems, but do we need to start looking at incorporating these into our quality measurement as well? I think we certainly do.

It's a nuanced question, there are not easy answers to it, but this is where our next studies are going to take us into starting to explore these a little further.

Dr Puri. I agree with you and I think that's an important point, because not one of us in this room will disagree that we think surgery is the appropriate treatment for stage I lung cancer, but as soon as you move out of this meeting, that is under debate right now. And I feel that the more upfront we can be about both short- and long-term outcomes after our best operations, the better evidence we are equipped with to face criticism of what we do every day for stage I lung cancer.

I would like to thank the committee for the opportunity to discuss this important paper.

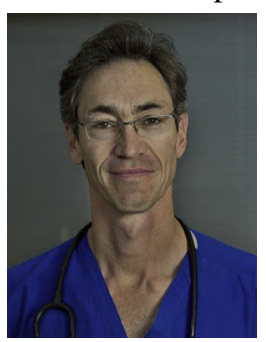

Dr Joseph B. Shrager (Stanford, Calif). Do you have data on the type of operation, anatomic versus nonanatomic in particular, at the high- versus low-performing centers, because you could perhaps understand why you might have better short-term morbidity but worse outcome if people are doing more wedge resections at certain centers?

Dr Fernandez. We do have that. This was a 6-minute presentation, it was hard to include all of those data, but there were essentially an equal number of anatomic resections at each center. Now, the higher performing centers had more segmentectomies and there were a little more lobectomies at the below average programs, but in terms of anatomic resections, they were more or less the same.

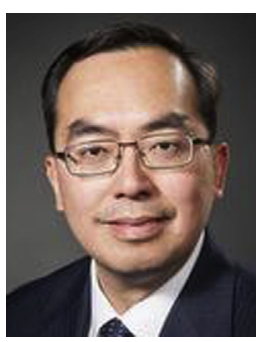

Dr Paul C. Lee (New Hyde Park, NY). I enjoyed your talk very much. By your conclusion, are you implying that as far as beyond surgical results there might be some differences maybe in medical oncology support, adjuvant chemotherapies? Have you looked at pathologic stage II or III patients and do you see a difference in those programs, because obviously most of the patients may not recur and you may not see the difference in stage II and stage III patients in judging the quality of the oncology support in the hospital.

Dr Fernandez. That's an important question. We haven't done that yet. We wanted to start with a clean analysis of surgical therapy and that's why we selected pathologic stage I lung cancer, but I can see how looking at stage II and stage III would be of interest as well in looking at other system factors. 


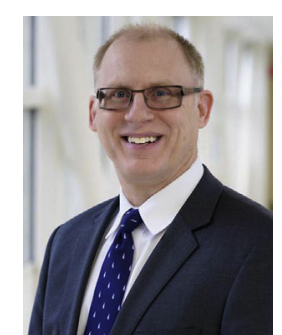

Dr Todd L. Demmy (Buffalo, NY). Have you looked at the low performing early outcome indicators for patients doing well in the long term to see what might be the driving difference? For instance, we run a relatively busy lung program through a small hospital. Postoperative bronchoscopy and other minor procedures that go to the OR to adjust for capacity issues in the endo suite getting tagged as "returned to the OR" complication as a bad outcome. Yet the patients go home in 2 to 3 days by being this aggressive with their management. That concerns me with the STS database coding. Could you tease it out and see what was the mechanism for having good long-term outcomes despite suboptimal shortterm metrics?

Thank you.

Dr Fernandez. Mortality drives the short-term performance more than anything else, operative mortality, and there was not a lot of variability in short-term outcomes where there was a lot of variability in the longer-term outcomes. We tried to look at a lot of different things: extent of resection, operative approach, lymph node staging. There was no predictor we could find to sort out why a high performing, short-term program would be low performing in the long-term or vice versa. That's something we were not as yet able to tease out with this data set.

Dr Demmy. I was saying, just to clarify, that all people who go back to the OR are tagged as a major complication that brings down your star value. I was wondering if you looked at that specifically.

Dr Fernandez. We have, and in the data field is actually unexpected return to the operating room, and it is institution-dependent how that would be coded. That's nothing we have specifically investigated.

Unidentified Speaker. Felix, a great talk. Since you were able to connect Medicare with the STS database, have you looked at other geographic or socioeconomic aspects of the various reporting centers? I know that is a different set of variables, but look at center level issues that might help explain some of the increased variability in the long-term issues whereas the short-term issues are going to be more reporting surgeon-specific? I didn't know if you had a chance to look at any of those variables.

Dr Fernandez. Yes, we would like to. We don't have that information available to us at present, but it would be valuable to look at.

Thank you. 
APPENDIX E1. IDENTIFICATION OF PROCEDURES FOR STAGING LUNG CANCER (CARRIER FILES, 2009-2013)

\begin{tabular}{|c|c|c|}
\hline Test category & Test & CPT/HCPCS codes \\
\hline Chest CT imaging & $\begin{array}{l}\text { Chest CT imaging (without contrast; with contrast; with and without } \\
\text { contrast) } \\
\text { Chest CT angiography }\end{array}$ & $\begin{array}{l}71250 ; 71260 ; 71270 \\
71275\end{array}$ \\
\hline Brain imaging & $\begin{array}{l}\text { Head/brain CT imaging (without contrast; with contrast; with and without } \\
\text { contrast) } \\
\text { Head MRI (without contrast; with contrast; with and without contrast) } \\
\text { Brain MRI, including brain stem (without contrast; with contrast; with and } \\
\text { without contrast) }\end{array}$ & $\begin{array}{l}70450 ; 70460 ; 70470 \\
70544 ; 70545 ; 70546 \\
70551 ; 70552 ; 70553\end{array}$ \\
\hline PET imaging & $\begin{array}{l}\text { PET imaging (limited; skull base to thigh; whole body) } \\
\text { PET/CT (limited; skull base to thigh; whole body) }\end{array}$ & $\begin{array}{l}78811 ; 78812 ; 78813 \\
78814 ; 78815 ; 78816\end{array}$ \\
\hline Invasive mediastinal staging & $\begin{array}{l}\text { EUS imaging } \\
\text { EUS imaging with FNA } \\
\text { Esophagogastroduodenoscopy with EUS imaging } \\
\text { Esophagogastroduodenoscopy with EUS imaging and FNA } \\
\text { GI EUS, interpretation only } \\
\text { Bronchoscopy with TBNA } \\
\text { Endobronchial ultrasound imaging during bronchoscopic diagnostic/ } \\
\text { therapeutic procedures } \\
\text { Mediastinotomy (cervical approach; transthoracic approach) } \\
\text { Mediastinoscopy including biopsy when performed } \\
\text { Thoracoscopy, lungs or plural space, without biopsy } \\
\text { Thoracoscopy of mediastinal space without biopsy } \\
\text { Thoracoscopy, lungs or plural space, without biopsy } \\
\text { Video-assisted thoracoscopy, lungs, pericardial sac, mediastinal or plural } \\
\text { space, without biopsy } \\
\text { Video-assisted thoracoscopy, mediastinal space with biopsy }\end{array}$ & $\begin{array}{l}43231 \\
43232 \\
43259 \\
43242 \\
76975 \\
31629 \text { (additional nodes, } 31633 \text { ) } \\
31620 \text { (add-on code, listed along } \\
\quad \text { with primary procedure) } \\
39000 ; 39010 \\
39400 \\
32601 \\
32605 \\
32602 \\
32601 \\
32606\end{array}$ \\
\hline
\end{tabular}

$\overline{C P T / H C P C S}$, Current Procedure Terminology/Healthcare Common Procedure Coding System; CT, computed tomography; MRI, magnetic resonance imaging; PET, positron emission tomography; EUS, endoscopic ultrasound; FNA, fine needle aspiration; GI, gastrointestinal; TBNA, transbronchial needle aspiration. 
APPENDIX E2. CONSOLIDATED STANDARDS FOR REPORTING OF TRIALS FLOW DIAGRAM DETAILING CREATION OF COHORTS FOR SHORT- AND LONG-TERM ANALYSES. SIR, STANDARDIZED INCIDENCE RATIO

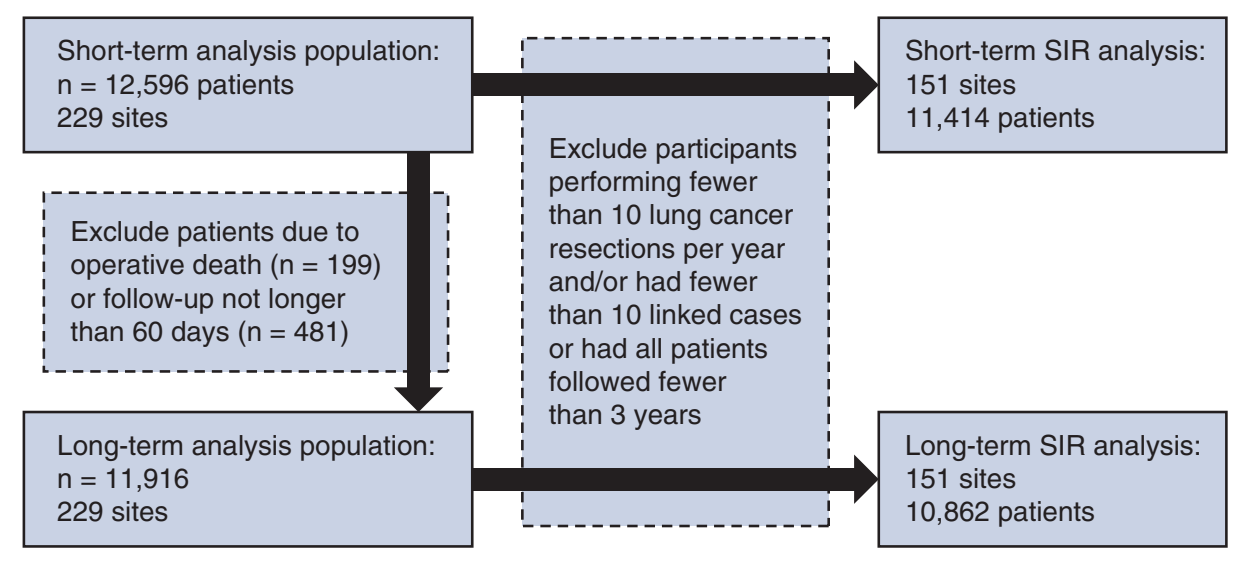




\section{APPENDIX E3. STANDARDIZED INCIDENCE RATIO ESTIMATION WITH BAYESIAN METHODOLOGY}

Participant (site/program)-specific SIR is the ratio between the participant's risk-adjusted rate and the risk-adjusted rate of a hypothetical average General Thoracic Surgery Database participant. The SIRs used in this article refer to freedom from an event. Hence, SIR $>1$ is consistent with better site performance (ie, more freedom from an event than expected on the basis of the patient case mix).

The STS participants (groups of patients within a particular program/site) are considered as random effects within the models to account for a participant-specific effect on patient outcomes (ie, clustering of patient outcomes within a participant). We estimated participant-specific SIRs within the Bayesian analysis framework because, in addition to its ability to consider participant-specific random effects, the Bayesian Markov Chain Monte Carlo simulations directly facilitate computation of credible intervals for the SIR estimates.

The concept of credible interval is similar to CI although within the Bayesian framework we can actually state that the true SIR value is within the $90 \%$ credible interval with $90 \%$ probability. The described Bayesian methodology has been used in site ratings within the STS database (eg, for the lobectomy composite). ${ }^{\mathrm{E} 1}$

We performed Markov Chain Monte Carlo simulations of model parameters using SAS software (PROC MCMC for short-term and PROC PHREG for long-term; SAS Institute). First, a long burn-in sequence of simulations $(50,000)$ was run to ensure convergence and then we obtained 1,000,000 simulations thinned to the final 5000 simulations by consideration of every 200th simulation to reduce autocorrelation within a chain of parameter estimates. The estimated participant-specific SIR values were computed as an average of the 5000 simulated values for this site; a $90 \%$ credible interval was obtained by considering the fifth (lower limit) and 95th (upper limit) percentiles of these 5000 simulations.

\section{E-Reference}

E1. Kozower BD, O’Brien SM, Kosinski AS, Magee MJ, Dokholyan R, Jacobs JP, et al. The Society of Thoracic Surgeons composite score for rating program performance for lobectomy for lung cancer. Ann Thorac Surg. 2016;101:1379-87. 